Pattern Recognition Letters

APRIL 2006; 27(6) : 658-666

http://dx.doi.org/10.1016/i.patrec.2005.10.004

(c) 2005 Elsevier B.V. All rights reserved
Archimer, archive institutionnelle de l'Ifremer http://www.ifremer.fr/docelec/

\title{
Automatic morphological detection of otolith nucleus
}

\author{
Frédéric $\mathrm{CaO}^{\mathrm{a}}$ and Ronan Fablet ${ }^{\mathrm{b}, *}$ \\ aINRIA/IRISA, Campus de Beaulieu, 35042 Rennes Cedex, France \\ ${ }^{\mathrm{b}}$ IFREMER/LASAA, BP 70, 29280 Plouzane, France \\ *: Corresponding author : Fax: +33 2982246 53; rfablet@ifremer.fr
}

\begin{abstract}
:
This paper deals with the automation of the acquisition of age and growth data from the analysis of otolith images. One of the key component of such a system is the automatic detection of the growth center, which serves as the basis for further processing (2D ring segmentation, age and growth estimation, ...). To this end, we develop a new and well-founded approach combining a morphological analysis of image level sets to the a contrario detection principle. This approach leads to a robust parameterless scheme, whose efficiency is demonstrated by an evaluation carried out for a set of several hundred of plaice otoliths.
\end{abstract}

Keywords: Mathematical morphology; A contrario detection; Otolith imaging 


\title{
Automatic morphological detection of otolith nucleus
}

\author{
Frédéric Cao \\ Ronan Fablet \\ INRIA/IRISA \\ IFREMER/LASAA \\ Campus de Beaulieu \\ BP 70, 29280 Plouzane, France \\ 35042 Rennes Cedex, France \\ rfablet@ifremer.fr \\ fcao@irisa.fr
}

\begin{abstract}
This paper deals with the automation of the acquisition of age and growth data from the analysis of otolith images. One of the key component of such a system is the automatic detection of the growth center, which serves as the basis for further processing (2D ring segmentation, age and growth estimation,...). To this end, we develop a new and well-founded approach combining a morphological analysis of image level sets to the a contrario detection principle. This approach leads to a robust parameterless scheme, whose efficiency is demonstrated by an evaluation carried out for a set of several hundred of plaice otoliths.
\end{abstract}

\section{Problem statement}

Age and growth data from fish otoliths are key information for a broad range of issues in fisheries science: for instance, the determination of age-length keys from the interpretation of fish otoliths for marine stock assessments [13] or the analysis of fish otolith growth patterns for marine ecology [8, 13]. These applications require large collections of age and growth data to carry out a consistent analysis. The acquisition of age and growth information is routinely performed by experts from the interpretation of concentric growth rings observed on fish otoliths as illustrated by Fig. 1 for a plaice otolith. Typically, several thousands of fish otoliths per year, species and stock are routinely interpreted by experts for marine stock assessment issues. Worldwide, it is currently estimated that several millions of otoliths are routinely interpreted each year for fisheries management issues. Given the associated interpretation cost, automation and quality assurance are major issues and digital imaging systems are of key interest through the creation 
of bases of otolith images. Hence, the development of computer-aided or automated tools for the interpretation of otolith images $[16,6,12]$.

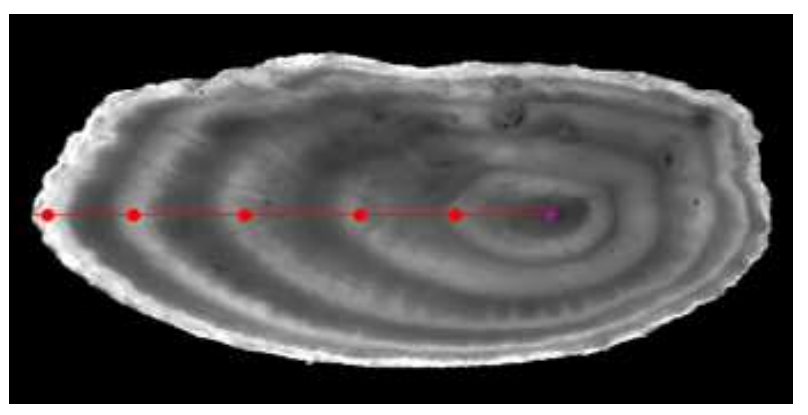

Figure 1: Illustration of plaice otolith interpretation for a 4 year old individual : image of the plaice otolith acquired under a binocular with transmitted light. The interpretation of the winter translucent rings is displayed by the markers set on the radial drawn on the main reading axis.

The automatic determination of the otolith growth center is of key importance both for ageing automation and computer-assisted otolith interpretation since it serves as the reference point for further analysis (automated extraction of interpretation axis, 2D ring segmentation, age and growth estimation,...). However, previous work targeted at otolith imaging $[4,7,14,17,19]$ has mainly focused on ring detection and segmentation issues, generally assuming that the otolith nucleus (also referred as the otolith center in the subsequent) is known. The detection of otolith nucleus has only been considered in [7, 19]. The solution proposed in [19], which simply resorts to the detection of the darkest point within a predefined area of interest set a priori, is not robust. In [7], the detection of the otolith center is a byproduct of the extraction of 2D growth rings. Though interesting, it involves an overcomplex parametrization of the underlying multi-agent system, which needs to be tuned appropriately. Conversely, in this paper, we design a robust and parameterless detection of otolith nucleus relying on a well-founded combination of morphological features to the $a$ contrario decision. The presentation and the evaluation of this new approach is organized as follows. Section 2 details the proposed morphological approach. Experiments, including a quantitative evaluation including a comparison to previously proposed approaches $[7,19]$ for a large set of plaice otoliths, are presented in Section 3. 


\section{Morphological nucleus detection}

\subsection{Topographic map}

In the following, we shall use the topographic map as a complete, contrast invariant representation of a grey level image. We briefly recall the main ingredients of this representation in this section.

Let $u$ be a grey level image. Its upper (resp. lower) level set at value $\lambda$ is $\chi_{\lambda}(u)=\left\{x \in \mathbb{R}^{2}, u(x) \geq \lambda\right\}$ (resp. $\left.\chi^{\lambda}(u)=\left\{x \in \mathbb{R}^{2}, u(x) \leq \lambda\right\}\right)$. It is a classical result of Mathematical Morphology [9, 15] that these level sets give a complete representation of $u$. Indeed, the two following reconstruction formulae hold:

$$
u(x)=\sup \left\{\lambda, x \in \chi_{\lambda}(u)\right\}=\inf \left\{\lambda, x \in \chi^{\lambda}(u)\right\}
$$

Moreover, this representation is invariant with respect to global contrast change. Indeed, if $v=g(u)$ where $g$ is an increasing continuous function, then $u$ and $v$ have the same family of level sets (obviously differently indexed). Conversely, if two images have the same family of level sets, then they differ by a monotone contrast change (possibly not continuous). Level lines are the topological boundaries of connected components of level sets, and the topographic map is the collection of all level lines. The topographic map also characterizes the image, up to an increasing contrast change. Since level sets are nested, they can be embedded in a tree structure, computed by an efficient algorithm due to Monasse and Guichard [11]. They call shape the interior of any level line. There are superior (resp. inferior) shapes, following whether they are obtained from an upper (resp. lower) level set. The denomination of shapes is due to the fact that they often correspond to shapes or part of shapes in real images [2]. This is consistent with the accepted fact that edges are normal to the gradient, and so locally follow level lines. For a smooth image, (say of class $C^{1}$ ), in the neighborhood of a point where the gradient is not equal to zero, a level line coïncide with an isophote (a line on which the grey level is constant). As a consequence, all level lines are everywhere parallel to edges, implying that edges are included in pieces of level lines. Now, contrary to edge points as given by Canny's detector [1], level lines are curves, and no connecting procedure is needed to retrieve curves.

\subsection{Nucleus detection}

Let us first remark that the otolith itself can be easily extracted as the largest shape in the image, that we shall denote by $S_{0}$. This is not very strict an hypothesis, since the acquisition of otolith images is well controlled, and the otolith is usually observed as it lies on a nearly constant background. Moreover, a few level lines still contain the greatest part 
of visual information. This can be easily checked (for instance) by quantizing the grey level at some few values. In about $99 \%$ of the cases we observed, the nucleus delimited by at least one level line. Other level lines give the position of growth rings, but they may be cut into a few parts. In fact, this is always the case in plaice otoliths, which exhibit a "blind part" containing nearly no structure. In order to detect the nucleus, we propose to select the best shape with respect to a few criteria.

Let us state the following qualitative facts: otoliths are vaguely elliptic, and nucleui correspond to a dark region close to the longest principal axis of the otolith. This region is also basically elliptic and no too excentric. From these qualitative description, we have to make quantitative arguments.

We proceed as follows: we start by removing shapes that definitely cannot be nucleus (it was checked on $100 \%$ of the observed images). We then introduce features measuring the previous qualitative properties. This will allow us to define a functional, and we will keep the shape that minimizes this functional. But contrarily to usual variational methods, this functional will have a quantitative meaning. It will tell how likely a shape will satisfy our geometric criteria only by chance. We will then see that it is possible to fix a robust automatic threshold to validate the detection. Besides, we will detail in section 3.2 a refinement of the proposed approach based on a priori information on nucleus position. The proposed approach is summarized in Fig.2.

\subsubsection{Maxima removal}

Let us first express the fact that the nucleus corresponds to a dark region. Let $\mathcal{E}_{0}$ be the shapes included in $S_{0}$ (that is, part of the otolith). We also denote by $\mathcal{E}_{1}$ the set of shapes in $\mathcal{E}_{0}$ that do not contain any superior shape. In other terms, shapes in $\mathcal{E}_{1}$ do not contain any local maxima. Of course, this is a bit strict, since noise creates lots of them. Thus, we first apply a grain filter $[10,18]$ to the image, which consists in removing shapes whose area is less than a given threshold. This scale parameter is the unique parameter of our method. Its value is not sensitive since the size of the nucleus is always of few hundreds pixels. The size of the grain filter was set to the same value 400 for all our experiments. Grain filter and maxima elimination leave of few dozens candidate shapes.

\subsubsection{Principal axis proximity}

Let us now denote by $\Delta$ the first principal axis of the otolith. Let $D$ be a perpendicular axis to $\Delta$, and $\left(X_{i}\right)_{i \in I}$ be a finite family of connected compact sets. Let $X$ be one of the $X_{i}$. We say that $X$ has a minimal distance to $\Delta$ on $D$ if 


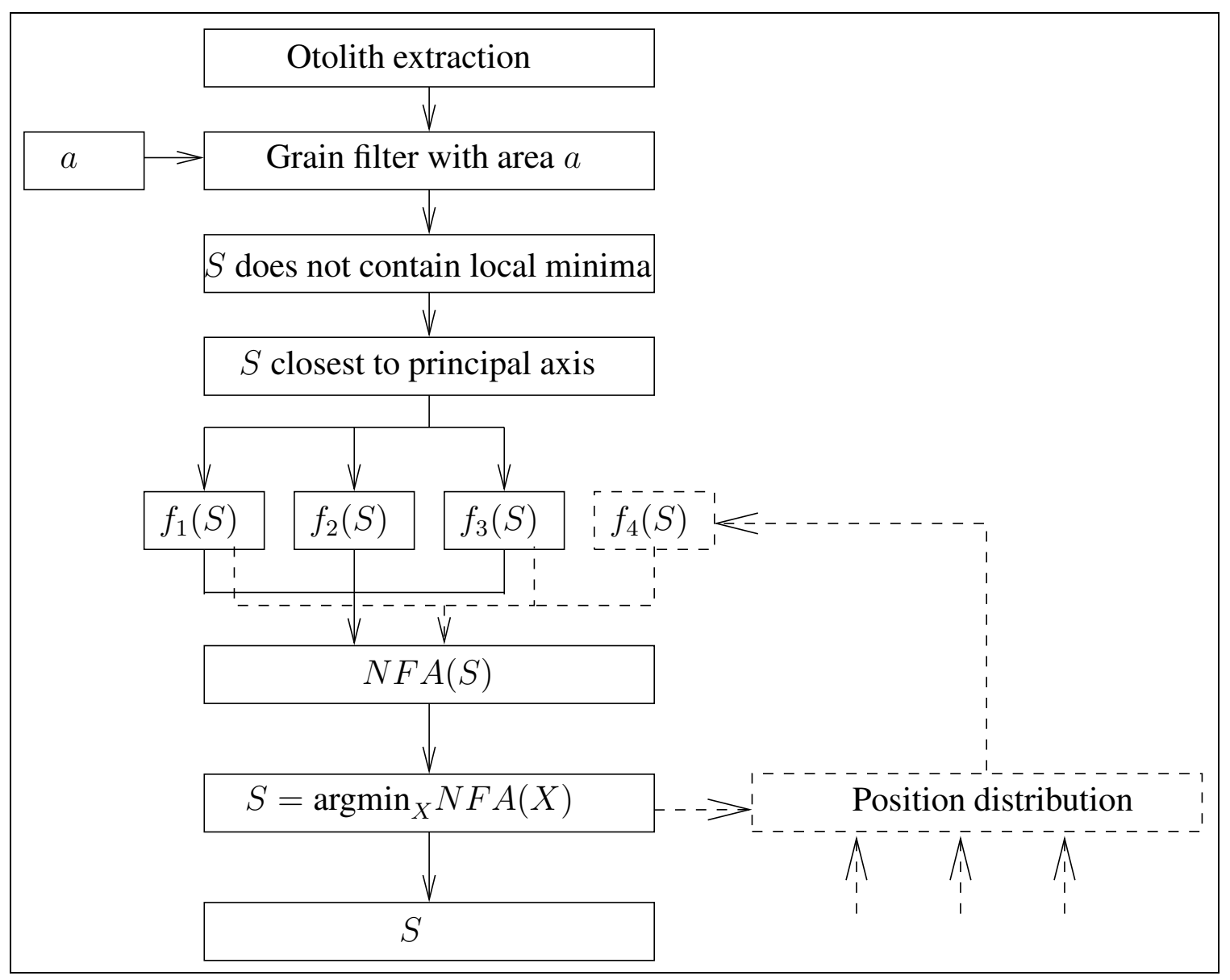

Figure 2: Diagram of the nucleus detection algorithm. The first step is the otolith extraction. Local extrema are filtered out by a grain filter with area parameter $a$. Shapes that contain local maxima or that are not the closest to the principal axis cannot be the nucleus. (This is only heuristic, but was satisfied for nearly all the images of the database.) Then three features $f_{1}, f_{2}, f_{3}$ allow to compute the NFA (Number of False Alarm). The best candidate is the one with the lowest NFA. In dashed lines, an optional loop: the position of the detections for a large database can be learned. It permits to propose a refinement of the proposed method using a feature $f_{4}$ which is more accurate than $f_{2}$, and leads to another NFA which experimentally gives better result. 
and only if $D \cap X \neq \emptyset$ and

$$
d(D \cap \Delta, D \cap X)=\min _{i \in I} d\left(D \cap \Delta, D \cap X_{i}\right) .
$$

In (2), we measure the distance between the point $(D \cap \Delta)$ and the set $\left(D \cap X_{i}\right)$. This condition simply means that if we restrict the observation on $D$, then $X$ is the closest to $\Delta$ among the $x_{i}$.

Finally, we say that $S \in \mathcal{E}$ if $S \in \mathcal{E}_{1}$ and if for any axis $D$, perpendicular to $\Delta$ such that $S \cap D \neq \emptyset$, then there exists $S^{\prime} \in \mathcal{E}_{1}$ such that $S^{\prime}$ has a minimal distance to $\Delta$ on $D$ among the shapes of $\mathcal{E}_{1}$ and $S \subset S^{\prime}$.

The interpretation is much simpler that this formal geometrical definition: basically, if we start from $\Delta$ and move along its normal, then the first dark shape that we encounter must contain $S$.

While the first step remove bright shapes, this second one removes elongated shapes that turn around the nucleus. (See Fig. 3.)

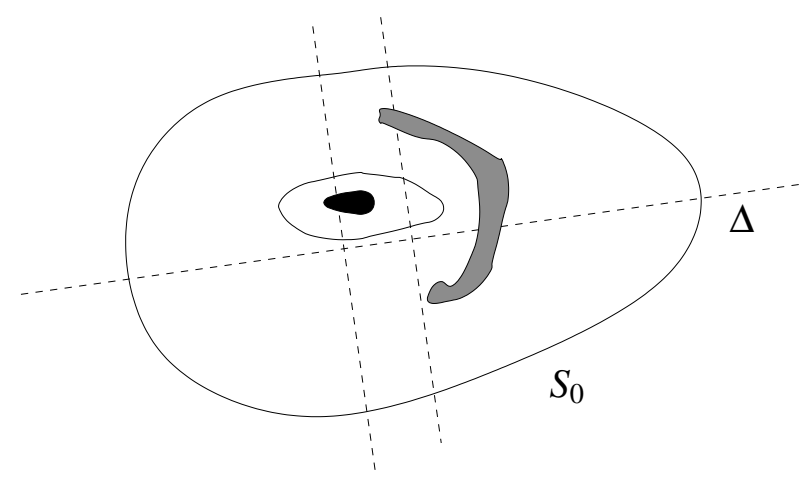

Figure 3: Proximity to principal axis. The black-filled shape is in $\mathcal{E}$ since in any direction orthogonal to $\Delta$, it is contained in the shape which is the closest to $\Delta$ (the white one). For the same reason, the white shape belongs to $\mathcal{E}$. On the contrary, one can find a normal to $\Delta$ such that the grey-filled shape is not the closest to $\Delta$ (since the white one is closer). Thus it does not belong to $\mathcal{E}$.

\subsubsection{Geometrical statistical selection}

The two preceding steps are only qualitative, and avoid any threshold. They are also quite general and experimentally very stable. We now aim at:

1. finding the best nucleus candidate.

2. giving a reliability measure to this detection. 
Let us define three functions $\left(f_{i}\right)_{i=1,2,3}$ representing the three geometrical qualities of a good nucleus. The first one is the area:

$$
f_{1}(S)=|S|
$$

The second one is:

$$
f_{2}(S)=\frac{|S|}{\max _{x \in S} d(x, \Delta)^{2}} .
$$

This function is scale invariant and measure how $S$ is close to $\Delta$, relatively to its size.

Let us now denote by $D\left(S, \Delta^{\perp}\right)$ the diameter of the projection of $S$ on the orthogonal direction to $\Delta$. We define

$$
f_{3}(S)=\frac{|S|}{D\left(S, \Delta^{\perp}\right)^{2}}
$$

This value is also dimensionless, and describes the "flatness" of $S$ with respect to the direction $\Delta$. Let us remark that a good nucleus candidate will simultaneously yield large values of the $f_{i}$, in the case our geometrical qualitative assumptions hold. However, fixing thresholds for the $f_{i}$ may be a numerically sensitive. To avoid this, let us make the a contrario hypothesis that the $f_{i}$ are statistically independent. This assumption is reasonable for any shape except the nucleus. Indeed, the size, the proximity to the axis and the flatness of the nucleus have a common cause, which is that the otolith grows in any direction from the nucleus. In classical hypothesis testing, this amounts to test the hypothesis $\mathcal{H}_{0}$ defined by "the $f_{i}$ are independent" against an alternative hypothesis $\mathcal{H}_{1}$ that they are not. The likelihood of $\mathcal{H}_{1}$ is not easy to compute, and requires a "good" model of otolith. In what follows, the decision will only be based on the null hypothesis $\mathcal{H}_{0}$. A natural way to detect this independence violation is as follows: let us fix some values $\lambda_{i}$ and compute, under the independence assumption, the expected number of shapes assuming values of the $f_{i}$ larger than $\lambda_{i}$. Whenever this value is lower than 1 and a shape $S$ satisfies $f_{i}(S) \geq \lambda_{i}$, then chance alone cannot explain the observation. This is related to the Helmholtz principle, introduced by Desolneux, Moisan and Morel [3]. The ideal situation would be to find a set of statistically independent features that give a complete description of what a nucleus should be. Since we are not capable to find such a precise model, we only keep three features, whose independence is plausible in a set or random shapes. For $i=1,2,3$, let us define $H_{i}$ the empirical inverse repartition function of $f_{i}$ :

$$
H_{i}(\mu)=\frac{\#\left\{S \in \mathcal{E}, f_{i}(S)>\mu\right\}}{\#\{S \in \mathcal{E}\}} .
$$

where \# denotes the cardinality.

Definition 1 Let $S \in \mathcal{E}$ and

$$
E_{1}(S)=\left(\max _{i=1,2,3} H_{i}\left(f_{i}(S)\right)\right)^{3} .
$$


We define the nucleus $N$ as the shape in $\mathcal{E}$ such that

$$
E_{1}(N)=\min _{S \in \mathcal{E}} E_{1}(S)
$$

Remark that $E_{1}$ is nothing but the empirical probability to observe the event "for all $i$, $f_{i} \geq f_{i}(S)$ ", under the $a$ contrario independence hypothesis. Thus, this definition means that the best candidate is the less probable under the independence assumption. In practice, we learn the histogram on a set of about 100 shapes, yielding positive marginals greater than $10^{-2}$. In practice, the minimizer of $E$ in our experiments assumes a value between $10^{-4}$ and $10^{-5}$. Remark that contrarily to Bayesian method where we look for the most probable candidate given a model, we detect the less probable candidate with respect to an a contrario independence assumption. Now, how can we assert that a probability is actually small? Otherwise said, can we find a natural threshold on the likelihood under the independence assumption? Let us first define number of false alarms.

Definition 2 We call number of false alarms (NFA) of $S$ the number

$$
N F A(S)=\# \mathcal{E} \cdot E_{1}(S)
$$

We also say that $S$ is $\varepsilon$-meaningful if $N F A(S)<\varepsilon$.

The additivity of expectation immediately gives the following result (see [3]).

Proposition 1 The expectation of the number of $\varepsilon$-meaningful shapes in the independence model is smaller than $\varepsilon$.

Proof. Let $X_{S}$ the binary variable equal to 1 if $S$ is $\varepsilon$-meaningful and 0 else. The expected number of $\varepsilon$-meaningful shapes is nothing but $\mathbb{E}\left(\sum_{S} X_{S}\right)$, where $\mathbb{E}$ denotes the expectation. By linearity,

$$
\mathbb{E}\left(\sum_{S} X_{S}\right)=\sum_{S \in \mathcal{E}} \mathbb{E}\left(X_{S}\right) .
$$

But $\mathbb{E}\left(X_{S}\right)$ is the probability that $S$ is meaningful. Let $Y_{i}=f_{i}(S)$. The a contrario assumption is that the $Y_{i}$ are independent, and their inverse repartition function is $H_{i}$. Then

$$
\begin{aligned}
P\left(X_{S}=1\right) & =P\left(\left(\max _{i} H_{i}\left(Y_{i}\right)\right)^{3}<\frac{\varepsilon}{\# \mathcal{E}}\right) \\
& =P\left(\forall i, H_{i}\left(Y_{i}\right)<\left(\frac{\varepsilon}{\# \mathcal{E}}\right)^{1 / 3}\right) \\
& =\prod_{i=1}^{3} P\left(H_{i}\left(Y_{i}\right)<\left(\frac{\varepsilon}{\# \mathcal{E}}\right)^{1 / 3}\right) \text { by independence } \\
& \leq \frac{\varepsilon}{\# \mathcal{E}} .
\end{aligned}
$$


The last inequality follows $P\left(H_{i}\left(Y_{i}\right)<t\right) \leq t$, because $H_{i}$ is the inverse repartition function of $Y_{i}$. Thus, the expectation in Eq.10 is a sum of $\# \mathcal{E}$ terms, all less than $\frac{\varepsilon}{\# \mathcal{E}}$. Hence, it is less than $\varepsilon$.

As a consequence, let us choose $\varepsilon<1$ and assume that we observe that a shape has a NFA less than $\varepsilon$. We know that, in average, there is less than one such a shape under the a contrario hypothesis. We conclude that the independence is certainly false and detect the shape as meaningful. Since in practice, we obtain NFA of magnitude $10^{-2}$, this means that we can expect $1 \%$ false alarms, which is coherent with the empirical results.

\section{Experimental results and refinement of the proposed approach}

\subsection{Results}

We first display two examples of the detection of the nucleus within otolith images for plaice samples. Fig. 4 reports these two detections, respectively for a 3 years and a 10 years old otolith. The red line outlines the shape selected by our morphological approach, the blue star the estimated position of the nucleus and the magenta circle its actual position manually pointed out. The second row zooms in around the otolith center. In both cases, the selected shape is actually located within the first translucent ring. Consequently, an accurate estimation of the position of the otolith center is deduced. An important feature stresses by these two examples is the robustness of the morphological detection to the increase in image complexity in terms of the number of concentric seasonal rings.

We have carried out an evaluation of the proposed technique on a set of 250 images of plaice otoliths from age groups 1 to 13 . For each image, the position of the nucleus as well as the position of the first translucent ring have been manually pointed out by the expert to provide the ground truth. To achieve a quantitative analysis of the detection performance, we define the rate of correct detection w.r.t. the threshold value $\alpha$ as follows. Let is denote by $D$ the -distance between the detected nucleus and the actual one. Rather than straightforwardly setting a threshold on $D$, we prefer accounting for the variations of the width of the first translucent ring. This will lead to a more relevant accuracy measure. Hence, the detection of the otolith center is considered as correct w.r.t. $\alpha$ if distance $D$ is lower than $\alpha$ times the distance from the actual nucleus center to the first translucent ring. In average, the first translucent ring is about 100 pixels wide. Setting $\alpha$ to 0.3 then roughly comes to retain only the detections for which the distance to the actual position is below 30 pixels. Besides, for $\alpha=1$, it indicates whether or not the estimated position of the nucleus is within the first translucent ring. In actual applications such as computer-aided acquisition of bases of interpreted otolith images or ring segmentation for automated ageing [5, 17], a detection accuracy $\alpha$ below 0.3 is satisfactory. In 
3 year old otolith
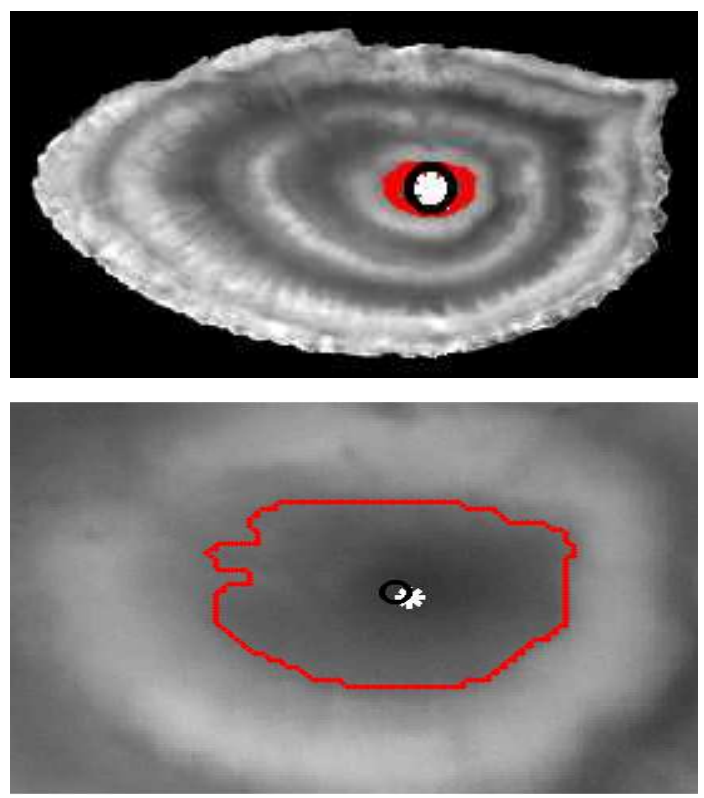

10 year old otolith
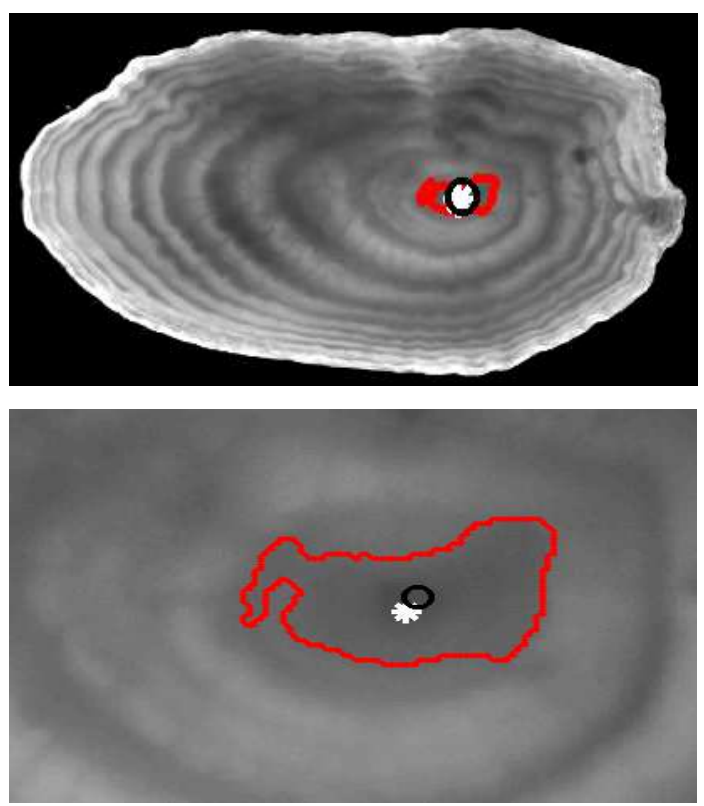

Figure 4: Two examples of the detection of otolith center using our morphological approach: the line superimposed to the image depicts the contour of the selected shape, marker $\circ$ (black) the estimated position of the otolith center, and marker $*($ white) its true position. The first row displays the image of the whole otolith, while the second row zooms in to the region close to the otolith center. 


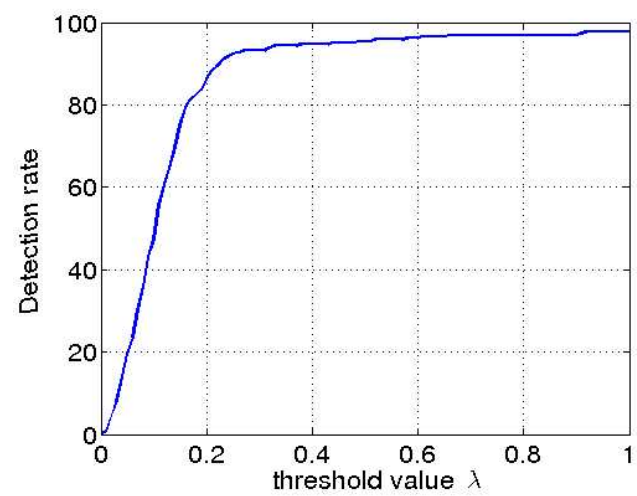

Figure 5: Detection performance on a set of 250 Plaice otolith images. We evaluate the rate of correct detection for a range of values of the threshold $\alpha$ (see text for details) using criterion (8).

the subsequent, this value will then be used as the reference value. Fig. 5 reports the results obtained using criterion (8). We obtain good results with an overall detection rate of $95.1 \%$ for a threshold value $\alpha$ of 0.3 .

\subsection{Detection refinement}

The use of criterion $E_{1}(N)$ (cf. Eq. 8), defined from purely geometrical features, leads to $2 \%$ misdetections outside of the first growth ring as illustrated by Fig. 7. For these samples, candidate areas corresponding to the nucleus however appear within the three best $E_{1}(S)$ scores. As detailed below, these results can be improved by a two-pass scheme. In order to improve the detection performances, additional information on the location of the nucleus can be exploited to refine its detection. This information is provided by the result of the method described above, and not by the user.

Otoliths are known to generally grow asymmetrically. As an illustration, Fig. 6 displays the distribution of the nucleus detections issued from our morphological detection. Note that, in general, the horizontal orientation of the otolith is unknown, i.e. we cannot distinguish the right from the left. We arbitrarily decide that the otolith is oriented such that the detected nucleus is at the right of the otolith center. The observed distribution is clearly unimodal and conveys relevant information to refine the nucleus detection. For instance, regarding this global distribution, some points appear as outliers likely to correspond to false detections.

We then perform a second pass as follows. Given the detections issued from criterion (8), we compute the median location of the extracted regions. This median position is exploited as an a priori prediction within our a contrario framework. It resorts to the introduction of feature within the geometrical statistical selection. This feature evaluates 


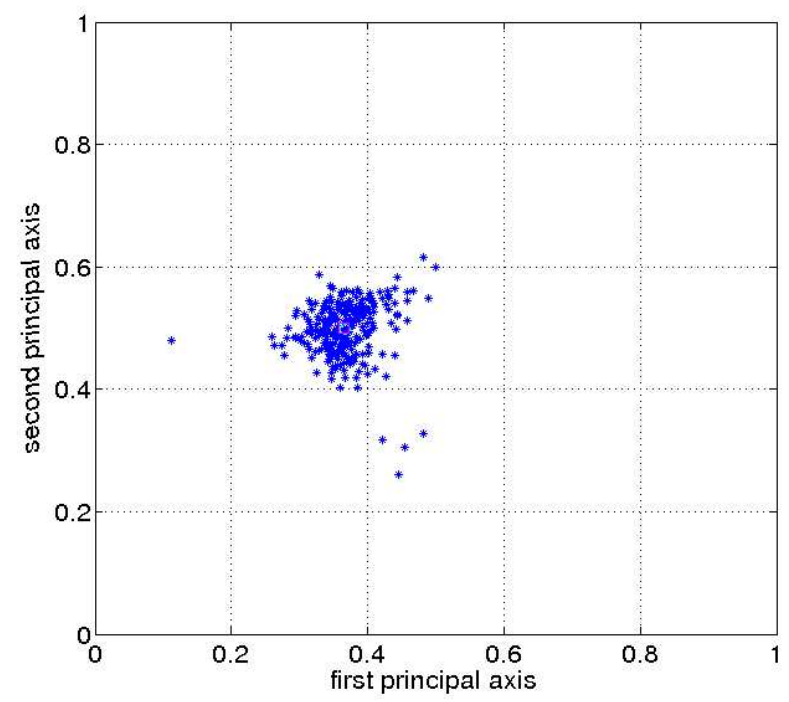

Figure 6: Distribution of the positions of the regions selected using criterion (8) for the processed dataset of right sagittae otoliths of plaice. The positions are computed in the frame of the principal axis of the otoliths, normalized w.r.t. the lengths of the otolith in these two directions, such that the selected region is at the right of the otolith center.

the distance between the center of the candidate region and the predicted position and is defined as follows:

$$
f_{4}(S)=-\left\|C_{S}-P\right\|
$$

where $P$ is the predicted nucleus position and $C_{S}$ the center of the candidate zone $S$. Given that, in general, the horizontal orientation of the otolith is unknown, $f_{4}(S)$ is computed as:

$$
f_{4}(S)=-\min \left(\left\|C_{S}-P_{L}\right\|,\left\|C_{S}-P_{R}\right\|\right)
$$

where $P_{L}$ and $P_{R}$ are respectively the predicted position of the nucleus towards the left and the right in the frame of the principal axis of the otolith. For this second pass, similarly to Eq. 8, we define the nucleus $N$ as the shape $S$ such that:

$$
E_{2}(N)=\min _{S \in \mathcal{E}} E_{2}(S) \text { with } E_{2}(S)=\left(\max _{i=1,3,4} H_{i}\left(f_{i}(S)\right)\right)^{3} .
$$

Let us stress that the considered criterion is not $\left(\max _{i=1,2,3,4} H_{i}\left(f_{i}(S)\right)\right)^{4}$. As a matter of fact, the a contrario principle relies on the detection of independence violations for the considered features, whereas $f_{2}(S)$ and $f_{4}(S)$ are obviously dependent. On the contrary, for a random set of shapes, the independence between $f_{1}(S), f_{3}(S)$ and $f_{4}(S)$ is a sound assumption, which permits to apply the a contrario detection.

Fig. 7 illustrates the gain brought by the proposed refinement. The use of criterion (13) further constrains the 

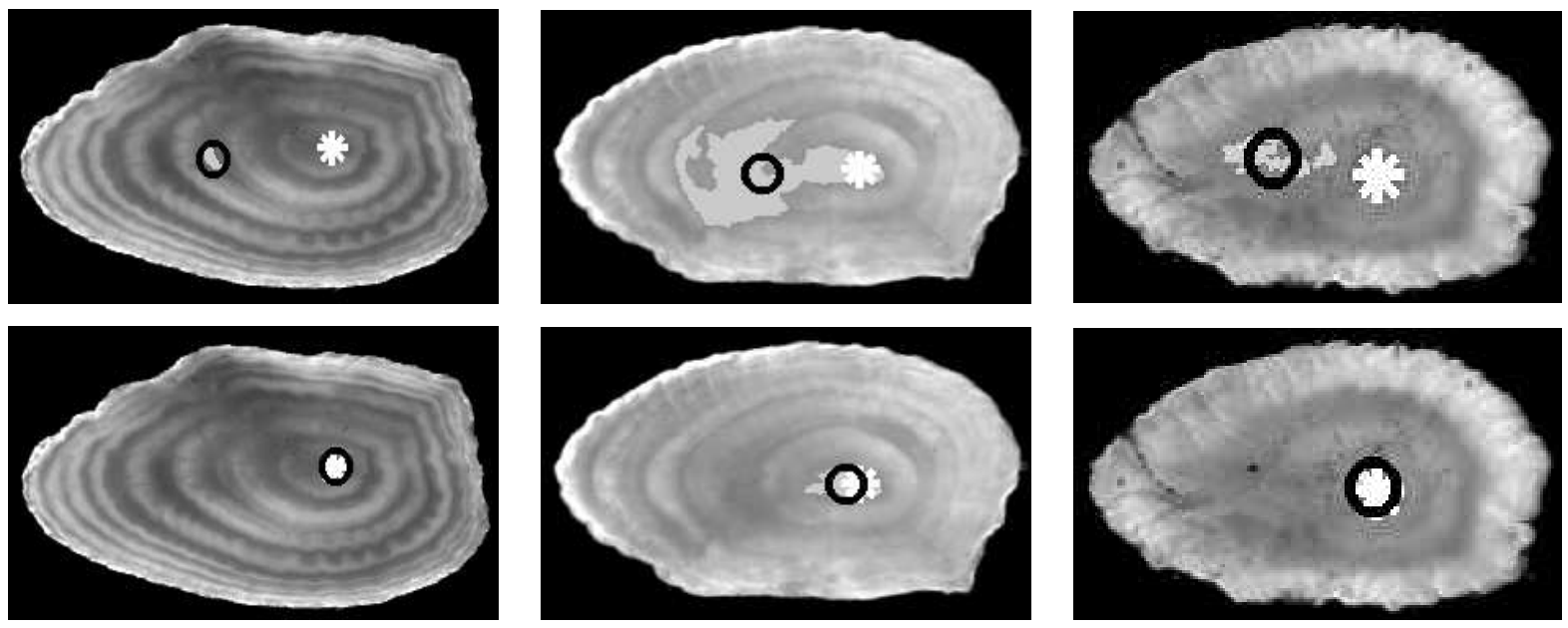

Figure 7: Refinement of the detection of the nucleus: the first row displays examples of misdetections using criterion (8) based on geometrical and photometric features; the second row reports the results obtained for same otoliths by the two-pass scheme using additional information on nucleus position. The shape $N$ selected as the nucleus shape (cf. Eq.8 is superimposed to the image as a gray region. Besides, marker $*$ (white) refers to the actual nucleus position, whereas marker $\circ$ (black) refers to the estimated one.

nucleus detection with some information on the position of the nucleus region to extract correct detections. These results are confirmed by the evaluation of the detection performance for the dataset of 250 plaice otolith images. We report in Fig. 8 the comparison of the one-pass and two-pass schemes. For a threshold value of 0.3 , the detection performances increase from $95.1 \%$ to $98.0 \%$. Besides, there are only $0.7 \%$ of false detections outside the first growth ring compared to $2.0 \%$ with the one-pass algorithm. We also report in Fig. 8 the histogram of the distances between the actual nucleus position and the estimated one, reported for the two-pass scheme: the mean distance equals 11.8 pixels (versus 15 pixels for the one-pass scheme) and $98.1 \%$ (versus $95 \%$ for the one-pass scheme) for the one-pass scheme of the detections are associated to a distance below 30 pixels. The results stress the improvement brought by the proposed refinement in terms of detection accuracy and demonstrate that we reach a satisfactory accuracy for actual applications. To further illustrate the practicability of the proposed approach, we report in Fig.9 a subset of the analyzed images with the associated detections.

\subsection{Comparison to previous work}

In order to stress the efficiency of our morphological approach, we have carried out an experimental comparison in detection accuracy w.r.t. previous ones proposed in [7] and [19]. Fig. 10 depicts the overall evaluation. Compared to previously proposed approaches, the morphological scheme largely outperforms the heuristic one used in [19], which 

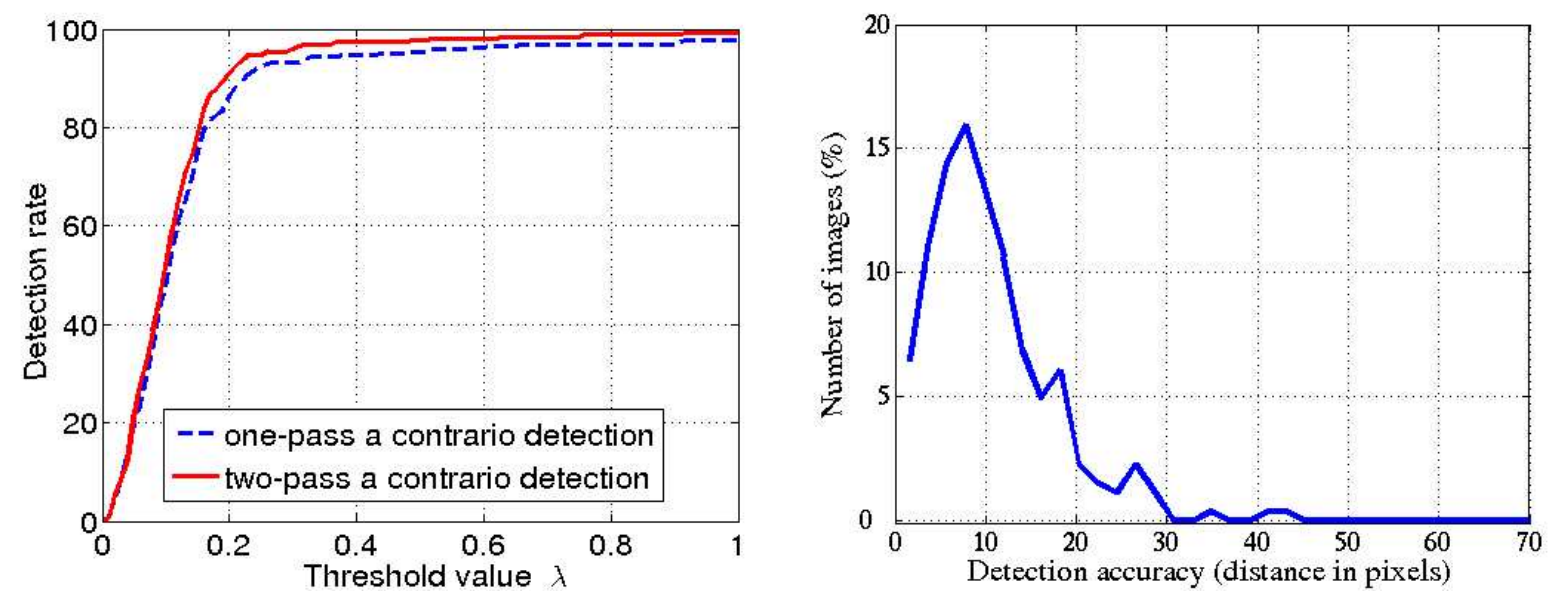

Figure 8: Detection performance on a set of 250 Plaice otolith images. The left plot displays the rates of correct detections for a range of values of the threshold $\alpha$ (see text for details) using the method proposed in this paper with (solid line) or without (dashed line) the use of a priori information on the nucleus position. The right plot depicts the histogram of the distances between the actual nucleus position and the estimated one for the two-pass scheme.

simply consists in retrieving the darkest point within a predefined window, and the multi-agent technique proposed in [7] (respectively, $98.1 \% 84 \%$ and $84 \%$ of correct detection for $\alpha=0.3$ ). Whereas multi-agent systems are known to involve quite complex parameterization, the proposed morphological method can be regarded as parameterless. It only requires to set the parameter value of the grain filter, used to filter out small regions. In our experiments, this value was set to 400 and the detection performances were shown to remain stable for a grain parameter within $[100,600]$.

\section{Conclusion}

We have developed a morphological approach for the detection of the nucleus region in otolith images. The reported evaluation and the comparison to previous work demonstrate the relevance of the chosen parameterless framework combining topographic representation of otolith images to an a contrario detection. In future work, we will further investigate this methodology to define robust and parameterless tools for extraction of geometrical structures in particular for the images of biological tissues depicting growth rings such as otoliths, tree trunks, coral sections, sea shells,.... Besides, the proposed method provides us with a key component to develop a fully automated system for the acquisition of age and growth data from otolith images. 

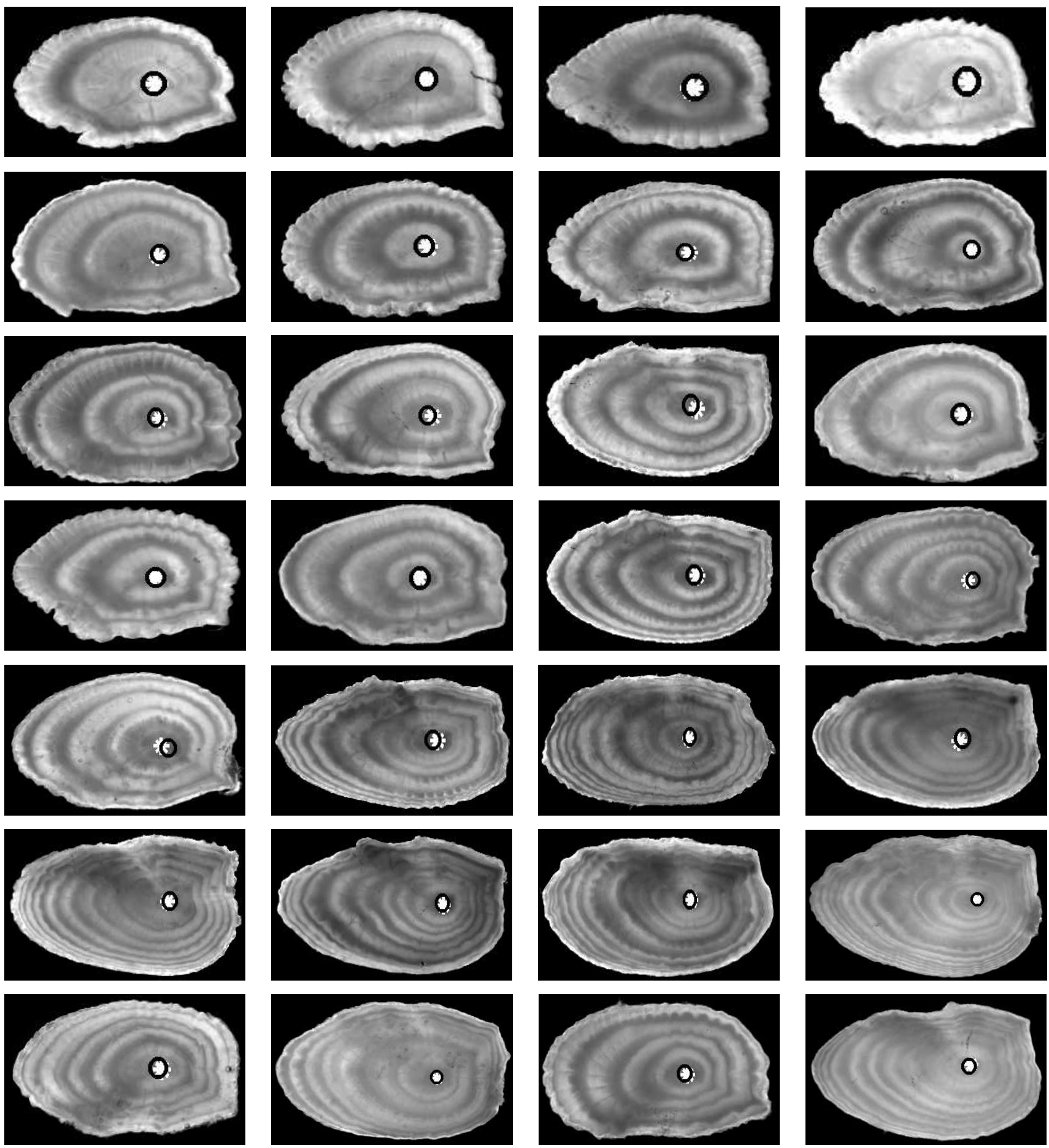

Figure 9: Illustration of the practicability of the two-pass scheme approach for a subset of the base of 250 otolith images. Marker * (white) refers to the actual nucleus position, whereas marker $\circ$ (black) refers to the detected one. 


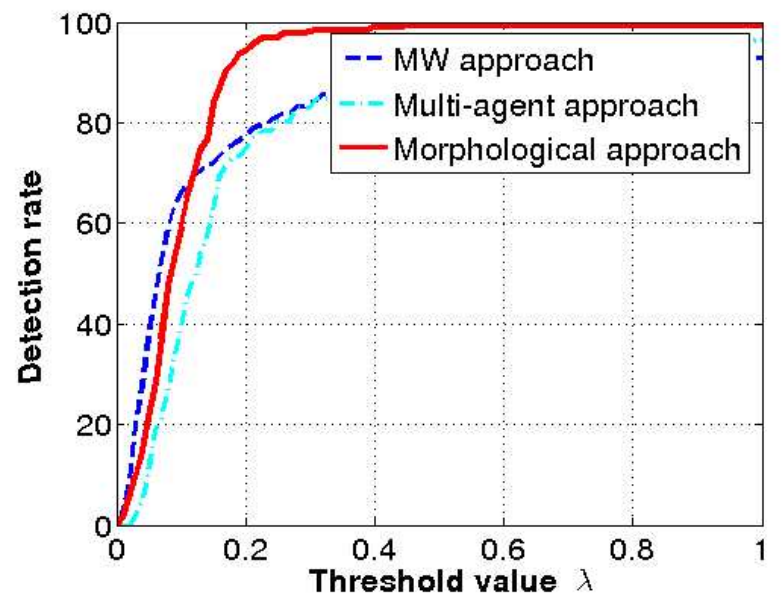

Figure 10: Detection performance on a set of 250 Plaice otolith images. We evaluate the rate of correct detection for a range of values of the threshold $\alpha$ (see text for details) for three techniques: the morphological approach presented in this paper (red line), the multi-agent technique introduced in [7] (blue - line) and the scheme used in [19] (cyan -. line). in terms of difference between the true and predicted positions of the otolith center. Markers + refer to the results supplied by the scheme used in [19], while the detections performed by our approach are displayed by markers o. The three methods need a CPU time about 1s per image.

\section{References}

[1] J. Canny. A computational approach to edge detection. IEEE Trans. on PAMI, 8(6):679-698, 1986.

[2] V. Caselles, T. Coll, and J.M. Morel. Topographic maps and local contrast changes in natural images. Int. J. of Comp. Vision, 33(1):5-27, 1999.

[3] A. Desolneux, L. Moisan, and J.M. Morel. A grouping principle and four applications. IEEE Trans. on PAMI, 25(4):508-513, 2003.

[4] R. Fablet, A. Benzinou, and C. Doncarli. Robust time-frequency model estimation in otolith images for fish age and growth analysis. In Proc. of 10th Int. Conf. on Image Processing, ICIP'03, Barcelona, Sept. 2003.

[5] Fablet, R. and Josse, N. L. 2005. Automated fish age estimation from otolith images using statistical learning. Fisheries Research 72(2-3): 279-290.

[6] R. Guay. Morphing technology applied to digital tree ring density measurement. In Int. Union of Forestry Org., Forest Products for Sustainable Forestry, Pullman, WA, 1997.

[7] A. Guillaud, A. Benzinou, H. Troadec, V. Rodin, and J. L. Bihan. Autonomous agents for edge detection and continuity perception on otolith images. Image and Vision Computing, 20(13-14):955-968, 2002. 
[8] J. Hare and R. Cowen. Effect of age, growth rate, and ontogeny on the otolith size - fish size relationship in bluefish, pomatomus saltatrix, and the implications for back-calculation of size in fish early life history stages. Can. Jal of Fish. Res. and Aqu. Sc., 52:1909-1922, 1997.

[9] G. Matheron. Random Sets and Integral Geometry. John Wiley N.Y., 1975.

[10] P. Monasse. Morphological Representation of Digital Images and Application to Registration. PhD thesis, Université Paris IX Dauphine, 2000.

[11] P. Monasse and F. Guichard. Fast computation of a contrast invariant representation. IEEE Trans. on Image Proc., 9(5):860$872,2000$.

[12] A. Morison, S. Robertson, and D. Smith. An integrated system for production fish aging: image analysis and quality insurance. North American Journal of Fisheries Management, 18:587-598, 1998.

[13] J. Panfili, H. de Pontual, H. Troadec, and P. Wright. Manual of Fish Sclerochronology. Eds Ifremer, 2003.

[14] S. Robertson and A. Morison. Development of an artificial neural network for automated age estimation. Technical Report 98/105, Marine and Freshwater Resources Institute, 1998.

[15] J. Serra. Image Analysis and Mathematical Morphology. Academic Press, 1982.

[16] G. Small and G. Hirschhorn. Computer-assisted age and growth pattern recognition of fish scales using a digitizing tablet. In G. H. R.C. Summerfelt, editor, Age and Growth of Fish, pages 397-410. ISU Press, 1987.

[17] H. Traodec, A. Benzinou, V. Rodin, and J. L. Bihan. Use of deformable templates for otolith 2D growth ring detection by digital image processing. Journal of Fisheries Research, 46(1-3):155-163, 2000.

[18] L. Vincent. Grayscale area openings and closings, their efficient implementation and applications. In J. Serra and P. Salembier, editors, Proc. of the $1^{\text {st }}$ Workshop on Math. Morphology and its App. to Sig. Proc., pages 22-27, 1993.

[19] H. Welleman and F. Storbeck. Automatic ageing of Plaice otoliths by means of image analysis. In D. Secor, J. Dean, and S. Campana, editors, Recent developments in Fish Otolith Research, pages 271-282. Univ. of South Carolina Press, 1995. 